\title{
Reliability-Based Control Design for Uncertain Systems
}

\author{
Luis G. Crespo * \\ National Institute of Aerospace, Hampton VA, 23666, USA \\ Sean P. Kenny ${ }^{\dagger}$ \\ Dynamic Systems and Control Branch, NASA LaRC, Hampton VA 23681
}

\begin{abstract}
This paper presents a robust control design methodology for systems with probabilistic parametric uncertainty. Control design is carried out by solving a reliability-based multi-objective optimization problem where the probability of violating design requirements is minimized. Simultaneously, failure domains are optimally enlarged to enable global improvements in the closed-loop performance. To enable an efficient numerical implementation, a hybrid approach for estimating reliability metrics is developed. This approach, which integrates deterministic sampling and asymptotic approximations, greatly reduces the numerical burden associated with complex probabilistic computations without compromising the accuracy of the results. Examples using output-feedback and full-state feedback with state estimation are used to demonstrate the ideas proposed.
\end{abstract}

\section{Introduction}

Achieving balance between stability and performance in the presence of uncertainties is one of the fundamental challenges faced by control engineers. Trade-offs must be made to

*Staff Scientist, 144 Research Drive, Hampton VA 23666, AIAA Professional Member

${ }^{\dagger}$ Aerospace Technologist, Dynamic Systems and Control Branch, NASA LaRC, Hampton VA 23681 
reach acceptable levels of stability and performance with adequate robustness to parameter uncertainty. These trade-offs are explicitly linked to the control engineer's choice of uncertainty model as well as how that model is exploited in the synthesis process. Usually, the assumed uncertainty model has a profound impact on the performance robustness of the closed-loop system.

Several uncertainty models, such as norm-bounded perturbations, interval analysis, fuzzy sets and probabilistic methods ${ }^{1,2}$ are typically used. The most commonly used robust control methods ${ }^{3}$ are $\mu$-synthesis and $H_{\infty}$. In these methods, uncertainty is modeled with norm-bounded complex perturbations of arbitrary structure about a nominal plant. This treatment is used primarily because it leads to a tractable set of sufficient conditions for robust stability, making the approach computationally efficient. These methods are based on the most pessimistic value of performance among the possible ones, usually referred to as 'worst-case'. This worst-case performance is usually realized only by a single member of the uncertain model set and by a particular input signal. No information is provided regarding the likelihood that this worst-case will ever occur in practice. In addition, the intrinsic mathematical requirements of the approach usually lead to conservative models of uncertainty, over-conservative designs and complicated compensators.

Probabilistic uncertainty not only defines a set of plants where the actual dynamic system is assumed to reside but also associates a weight, i.e., the value of the probability density function, to each member of the set. In contrast to conventional robust control methods, this 'additional dimension' allows the pursuit of robustly optimal solutions in the probabilistic sense. For instance, reliability-based design searches for solutions that minimize the probability of violating design requirements prescribed in terms of inequality constraints. Hence, reliability-based control design searches for the compensator that places as much probability as possible within the region where the design requirements are satisfied. Notice that this allows the search for the compensator with the best robustness for a given control structure, e.g. the most robust PID controller, even though the violation of some the design requirements for some of the plants in the uncertainty set is unavoidable.

Synthesis approaches based on random searches ${ }^{4-7}$ and stochastic gradient algorithms ${ }^{8,9}$ have been applied to probabilistic robust control. In these studies, random sampling is the primary tool for assessing and pursuing acceptable levels of robustness in the control solution. On the other hand, asymptotic approximations ${ }^{10-12}$ for the estimation of failure probabilities have been only used as a control analysis tool. This paper integrates these numerical tools. 
The main contributions of this paper are as follows:

1. The use of shapeable failure domains within the reliability formulation. This allows the concentration of the random outcome about regions where an improved controlled performance is attained.

2. The formulation of a unified framework where reliability metrics for random variables and processes are integrated.

3. The integrated use of deterministic sampling and asymptotic approximations in a hybrid approach. This approach (i) reduces the computational complexity of the synthesis algorithm without compromising the accuracy of the results, (ii) eliminates the random character of the estimation, and (iii) eliminates the high computational demands associated with the estimation of small failure probabilities via Monte Carlo sampling. These improvements mitigate the high computational demands of existing design strategies.

This paper is organized as follows. Section II presents basic concepts related with control and probabilistic uncertainty. Section III introduces the reliability metrics for random variables and processes to be used throughout the the paper. Realizations to stability, time- and frequency-dependent performance metrics are provided therein. Section IV presents the hybrid approach used for the estimation of the reliability metrics previously introduced. The reliability-based control synthesis procedure is presented in Section V, including robust performance considerations and the synthesis algorithm. Two examples are presented in Section VI, where a satellite's attitude control problem and the disturbance rejection in a flexible beam are used to demonstrate the method. Finally, some conclusions are stated in Section VII.

\section{System Dynamics}

Let $\mathbf{p}$ be a vector of random variables used to model the uncertain parameters of the system. In this study, $\mathbf{p}$ is prescribed a priori by the joint probability density function $(\mathrm{PDF}) f_{\mathbf{p}}(\mathbf{p})$ or equivalently by the cumulative distribution function $(\mathrm{CDF}) F_{\mathbf{p}}(\mathbf{p})^{\mathrm{a}}$. The set of values that $\mathbf{p}$ could take, called the support of $\mathbf{p}$, will be denoted as $\Delta_{\mathbf{p}}$.

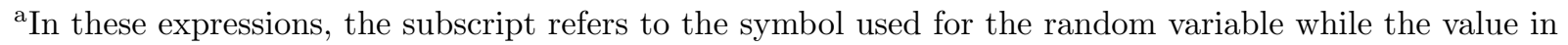
parenthesis refers to a particular realization.
} 
Consider the probabilistic model $\mathcal{M}(\mathbf{p})$ of a Linear Time Invariant (LTI) system, where the dependence of the model on the uncertain parameters could be non-linear. The reader must notice however, that the developments presented herein do not require the system to be LTI. The propagation of $\Delta_{\mathbf{p}}$ through $\mathcal{M}$ leads to a set of uncertain plant models in which the physical system is assumed to reside. The probability of occurrence of a plant within this set is fully determined by $\mathcal{M}$ and $\mathbf{p}$. In a transfer function representation, we will refer to $G(\mathbf{p})$ as the uncertain plant and to $K(\mathbf{k})$ as the compensator, where $\mathbf{k}$ is the vector of design parameters to be determined. Alternatively, a state space realization of $\mathcal{M}(\mathbf{p})$ leads to

$$
\begin{aligned}
& \dot{\mathbf{x}}=\mathbf{A}(\mathbf{p}) \mathbf{x}+\mathbf{B}(\mathbf{p}) \mathbf{u}+\mathbf{F}(\mathbf{p}) \mathbf{z} \\
& \mathbf{y}=\mathbf{C}(\mathbf{p}) \mathbf{x}+\mathbf{D}(\mathbf{p}) \mathbf{u}+\mathbf{E}(\mathbf{p}) \mathbf{v}
\end{aligned}
$$

where $\mathbf{x}$ is the state, $\mathbf{u}$ is the control, $\mathbf{z}$ is process noise, $\mathbf{y}$ is the system output and $\mathbf{v}$ is sensor noise. The noise signals are commonly modeled as delta correlated Gaussian white noises satisfying $\mathrm{E}[\tilde{\mathbf{z}}]=\mathbf{0}$ and $\mathrm{E}\left[\tilde{\mathbf{z}}(t) \tilde{\mathbf{z}}^{T}(t+\tau)\right]=\mathbf{S} \delta(\tau)$, where $\tilde{\mathbf{z}}=\left[\mathbf{z}^{T}, \mathbf{v}^{T}\right]^{T}$ and $\mathbf{S}$ is a constant spectral density matrix. In what follows, the explicit dependence on $\mathbf{p}$ is omitted while $\mathbf{D}$ is assumed to be zero.

As a result of uncertainty, important properties used in control design do not hold due to the offset between the deterministic mathematical model and the actual dynamic system. The effects of parametric uncertainty on the Separation Principle are considered next. For the full-state feedback law $\mathbf{u}=-\mathbf{G} \hat{\mathbf{x}}$ and a full-order observer with gain $\mathbf{L}$ based on the expected plant $\mathrm{E}[\mathcal{M}(\mathbf{p})]$ (any other deterministic plant such as $\mathcal{M}(\mathrm{E}[\mathbf{p}]$ ) could be used instead), the closed-loop dynamics is given by

$$
\begin{gathered}
\dot{\tilde{\mathbf{x}}}=\tilde{\mathbf{A}} \tilde{\mathbf{x}}+\tilde{\mathbf{B}} \tilde{\mathbf{z}} \\
\tilde{\mathbf{y}}=\tilde{\mathbf{C}} \tilde{\mathbf{x}}+\tilde{\mathbf{E}} \tilde{\mathbf{z}} \\
\tilde{\mathbf{A}}=\left[\begin{array}{c|c}
\mathbf{A}-\mathbf{B G} & \mathbf{B G} \\
\hline \mathbf{A}-\mathrm{E}[\mathbf{A}]+(\mathrm{E}[\mathbf{B}]-\mathbf{B}) \mathbf{G} & (\mathbf{B}-\mathrm{E}[\mathbf{B}]) \mathbf{G}+\mathrm{E}[\mathbf{A}]-\mathbf{L E}[\mathbf{C}] \\
+\mathbf{L}(\mathrm{E}[\mathbf{C}]-\mathbf{C})
\end{array}\right]
\end{gathered}
$$




$$
\tilde{\mathbf{B}}=\left[\begin{array}{c|c}
\mathbf{F} & \mathbf{0} \\
\hline \mathbf{F} & -\mathbf{L E}
\end{array}\right]
$$

where $\tilde{\mathbf{x}}=\left[\mathbf{x}^{T}, \mathbf{e}^{T}\right]^{T}$ is the augmented state vector, $\mathbf{e}=\mathbf{x}-\hat{\mathbf{x}}$ is the estimation error, $\hat{\mathbf{x}}$ is the estimation of $\mathbf{x}, \tilde{\mathbf{C}}=\left[\mathbf{C}^{T} \mid \mathbf{0}^{T}\right]^{T}$ and $\tilde{\mathbf{E}}=\left[\mathbf{0}^{T} \mid \mathbf{E}^{T}\right]^{T}$. The vector $\mathbf{k}$ is formed by the feedback gain $\mathbf{G}$ and the observer gain $\mathbf{L}$. Notice that the Separation Principle holds, i.e. $\tilde{\mathbf{A}}$ is upper triangular, if the deterministic plant used to generate the observer matches exactly the actual dynamic system. Uncertainty in the plant makes the Separation Principle unattainable. In addition, the random closed-loop poles do not occur at the locations selected for the full-state feedback, i.e. poles of the $\tilde{\mathbf{A}}_{1,1}$ subsystem, nor at the locations for the full-order observer, i.e. poles of the $\tilde{\mathbf{A}}_{2,2}$ subsystem.

\section{Reliability-Based Metrics}

The propagation of a fixed set of parameters of the plant through conventional control analysis tools leads to set of scalar quantities, e.g. closed loop poles, and a set of fields, e.g. step responses and Bode plots. The propagation of probabilistic uncertainty through the same tools leads to random variables, e.g. random closed-loop poles, and random processes, e.g. the step responses become random processes parameterized by time and the Bode plots become random processes parameterized by frequency. In this section we first introduce reliability metrics for random variables and processes. Such metrics will be used to quantify the violation of the design requirements. Specific realizations corresponding to stability, time and frequency requirements are then provided. In general, we will use $x$ and $x(h)$ to denote a random variable and a random process dependent on $\mathbf{p}$ through the plant model. For the random process $x(h), h$ refers to an arbitrary parameter such as time or frequency.

\section{A. Random Variables}

We start by introducing the concept of probability of failure. Let $x(\mathbf{p})$ be the random variable of interest and $x \leq \underline{x}$ be a design requirement. This event will be referred to as failure. The corresponding failure set is given by $\mathcal{F}=\{x \mid x \in(-\infty, \underline{x}]\}$, where the failure envelope $\underline{x}$ is a deterministic quantity prescribed in advance. The admissible domain, namely $\mathcal{A}=\{x \mid x \in$ $(\underline{x}, \infty)\}$, is the complement of the failure domain. The same type of discrimination can be done in the parameter space $\mathbf{p}$ by using $x(\mathbf{p})$. The function $g(\mathbf{p}, \underline{x})=x(\mathbf{p})-\underline{x}$, called the 
limit state function, divides the parameter space in two parts, the domain leading to $\mathcal{A}$, i.e. $g(\mathbf{p}, \underline{x})>0$, and the domain leading to $\mathcal{F}$, i.e. $g(\mathbf{p}, \underline{x}) \leq 0$. Hence, $\mathcal{F}$ results from mapping the set $\left\{\mathbf{p} \in \Delta_{\mathbf{p}} \mid g(\mathbf{p}, \underline{x}) \leq 0\right\}$ through $x(\mathbf{p})$. In this case, the probability of failure $P_{f}$ is given by

$$
P_{f}=\mathrm{P}[x \leq \underline{x}]=\int_{x \leq \underline{x}} f_{x}(x) d x=\int_{g \leq 0} f_{\mathbf{p}}(\mathbf{p}) d \mathbf{p}
$$

Similar expressions can be derived if the design requirement is $x \geq \bar{x}$. A reliability metric for $x$ in which constraints from below and above are present is given by

$$
r_{x}(\underline{x}, \bar{x}) \triangleq \underline{r}_{x}(\underline{x})+\bar{r}_{x}(\bar{x})
$$

where

$$
\begin{gathered}
\underline{r}_{x}(\underline{x}) \triangleq \mathrm{P}[x<\underline{x}]=F_{x}(\underline{x}) \\
\bar{r}_{x}(\bar{x}) \triangleq \mathrm{P}[x>\bar{x}]=1-F_{x}(\bar{x})
\end{gathered}
$$

Notice that $\underline{r}_{x}(\underline{x})$ is equivalent to Equation (5). We will refer to $\underline{x}$ and $\bar{x}$ as the envelopes of the failure domain $\mathcal{F}=\{x \mid x \in(-\infty, \underline{x}] \cup[\bar{x}, \infty)\}$. Notice that the under-bar and the over-bar refer to the bound from below and the bound from above of the admissible domain $\mathcal{A}=\{x \mid x \in(\underline{x}, \bar{x})\}$. This convention will be used for the reminder of the paper. Notice that the mapping of the corresponding limit state function through $x(\mathbf{p})$ leads to the failure envelope(s). Hence, there is a direct correspondence between $\mathcal{F}$ and $g$. A sketch with relevant information is provided in Figure 1.

\section{B. Random Processes}

The random process $x(h)$ can be considered as the parameterization of a random variable by the deterministic quantity $h$. The random process $x(h)$ is specified by the set of $\mathrm{CDFs}^{13}$ $F_{x(h)}(x, h)$. For instance, the system output $y(t)$ is prescribed by $F_{y(t)}(y, t)$. The evaluation of the process at a particular $h$ value, say $h_{i}$, leads to a random variable prescribed by $F_{x}(x)=F_{x(h)}\left(x, h_{i}\right)$. In general, the support and the percentiles of $x(h)$ depend on $h$. 


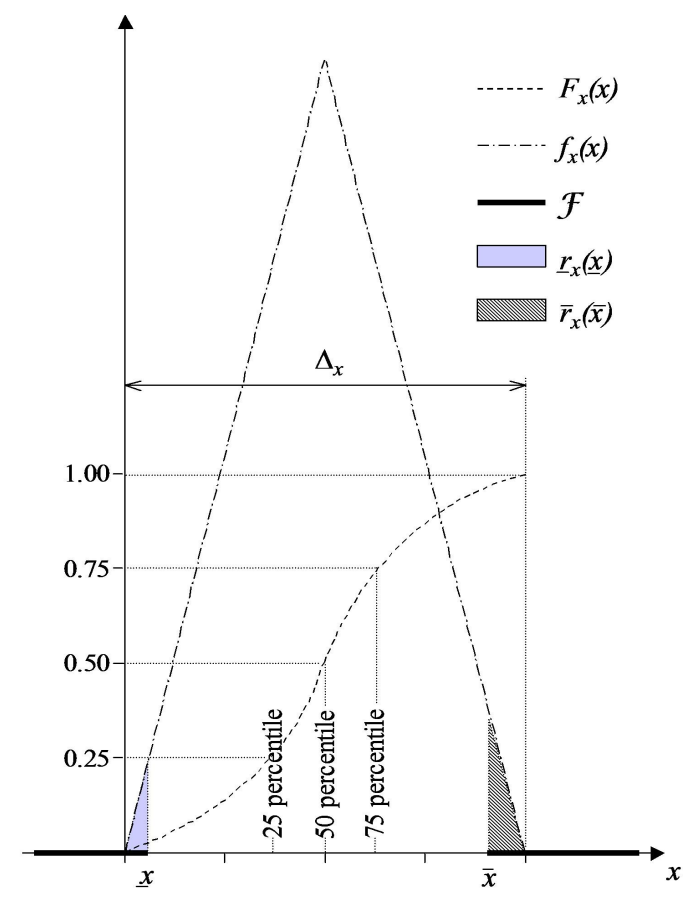

Figure 1. Sketch on the reliability metric for $x$.

In this context, a reliability metric for $x(h)$ is cast as follows

$$
r_{x(h)}(\underline{x}(h), \bar{x}(h)) \triangleq \underline{r}_{x(h)}(\underline{x}(h))+\bar{r}_{x(h)}(\bar{x}(h))
$$

where

$$
\begin{aligned}
& \underline{r}_{x(h)}(\underline{x}(h)) \triangleq \int_{h_{1}}^{h_{2}} \mathrm{P}[x(h)<\underline{x}(h)] d h=\int_{h_{1}}^{h_{2}} F_{x(h)}(\underline{x}(h), h) d h \\
& \bar{r}_{x(h)}(\bar{x}(h)) \triangleq \int_{h_{3}}^{h_{4}} \mathrm{P}[x(h)>\bar{x}(h)] d h=\int_{h_{3}}^{h_{4}} 1-F_{x(h)}(\bar{x}(h), h) d h
\end{aligned}
$$

are the costs of violating the constraints $x(h) \leq \underline{x}(h)$ and $x(h) \geq \bar{x}(h)$ respectively. The failure envelope functions, namely $\underline{x}(h)$ and $\bar{x}(h)$, are deterministic functions that delimit the failure domain $\mathcal{F}=\left\{(x, h) \mid\left(x(h) \leq \underline{\mathrm{x}}(h) \forall h \in\left[h_{1}, h_{2}\right]\right) \cup\left(x(h) \geq \bar{x}(h) \forall h \in\left[h_{3}, h_{4}\right]\right)\right\}$. Notice that the admissible domain $\mathcal{A}$ is bounded by $\underline{x}(h)$ from below and by $\bar{x}(h)$ from above. The reader shall realize that Equation (9) is a natural extension of Equation (6). A sketch with some of the pertinent metrics is provided in Figure 2. In the top plot, the 1, 25, 75 
and 99 percentiles $^{\mathrm{b}}$ are shown along with the linear failure envelopes $\underline{x}(h)$ and $\bar{x}(h)$. In the bottom plot, the integrands of Equations (10-11) corresponding to the configuration in the top plot are shown. Notice that if the process is contained within the set $\mathcal{A}$ the reliability
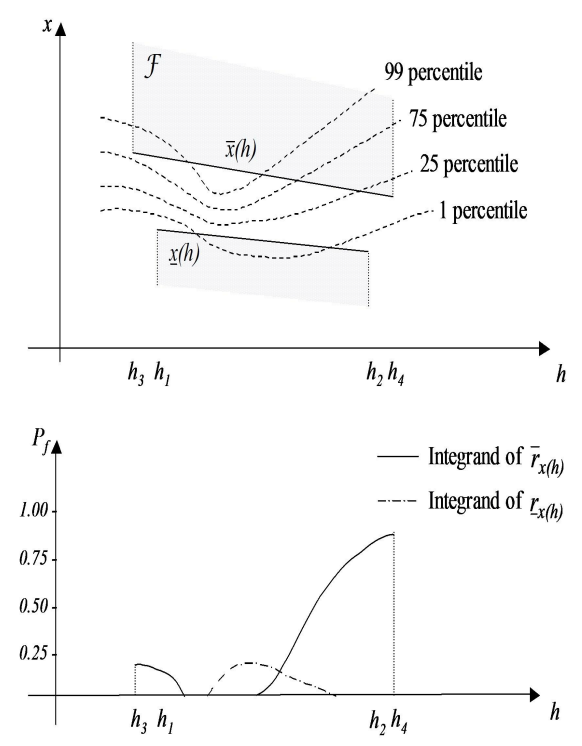

Figure 2. Sketch on the reliability metric for $x(h)$.

metric $r_{x(h)}$ is zero, meaning that the inequality constraints are satisfied for all parameter values in $\Delta_{\mathbf{p}}$.

\section{Realizations}

1. Robust Stability

A LTI system is robustly stable if all its poles are in the left hand side of the complex plane for all possible values of the random parameters. A reliability assessment of stability is given by

$$
\mathrm{P}\left[\bigcup_{i=1}^{v}\left(\Re\left[s_{i}\right]>0\right)\right]=\epsilon
$$

\footnotetext{
${ }^{\mathrm{b}}$ Recall that the $m$ percentile, given by the $x$ values satisfying $F_{x(h)}(x, h)=m / 100$, defines a line under which $m \%$ of the probability lies. These lines allow us the visualize the $h$ dependence of the PDF.
} 
where $s_{i}$ with $i=1,2, \ldots v$ is a random pole, $\Re[\cdot]$ is the real part operator and $\epsilon$ is the resulting probability of instability. Robust stability is attained if $\epsilon=0$. Stability can also be cast via

$$
\lambda \triangleq \max \left\{\Re\left[s_{1}\right], \Re\left[s_{2}\right], \ldots, \Re\left[s_{v}\right]\right\}
$$

In terms of $\lambda$, the probability of instability is given by $\bar{r}_{\lambda}(0)$. Robust stability is attained if $\bar{r}_{\lambda}(0)=0$.

Several comments are now pertinent. Reaching robust stability may not be feasible for the given support $\Delta_{\mathbf{p}}$ (even though it is bounded) and the assumed control structure $K(\mathbf{k})$. Notice also that the acceptance of a small non-zero probability of instability could be desirable from the performance point of view. For instance, by allowing the right low-probability tail of $f_{\lambda}(\lambda)$ to lie on the right hand side of the complex plane significant enhancements in the performance of the plants associated with the high probability portions of the PDF can be attained. Rather than advocating for the acceptance of the risk that this practice implies, we would like to highlight that by allowing small values of $\epsilon$, the trade-off between robustness and performance can be quantified.

\section{Time-Domain}

Quite frequently, performance requirements are prescribed in terms of time-domain specifications. The propagation of $f_{\mathbf{p}}(\mathbf{p})$ leads to random processes for the time responses. Denote with $x(t)$ an arbitrary random process with $\operatorname{CDF} F_{x(t)}(x, t)$. Such process is parameterized by time $t$ and the compensator design variable $\mathbf{k}$. The dependence of $x(t)$ on $\mathbf{k}$ has been

omitted for the sake of simplifying the notation. Reliability metrics for relevant processes can be cast using Equation (9). For instance, while settling time and overshoot requirements are integrated using $r_{y(t)}(\underline{y}(t), \bar{y}(t))$, the control saturation requirement $|u|<U$ leads to $r_{u(t)}(-U, U)$.

A reliability metric for assessing the effects of noise on the uncertain plant is formulated next. The state covariance matrix, defined as $\mathbf{Q}(t)=\mathrm{E}\left[\tilde{\mathbf{x}}(t) \tilde{\mathbf{x}}^{T}(t)\right]$, is given by the solution to the covariance equation

$$
\dot{\mathbf{Q}}=\tilde{\mathbf{A}} \mathbf{Q}+\mathbf{Q} \tilde{\mathbf{A}}^{T}+\tilde{\mathbf{B}} \mathbf{S} \tilde{\mathbf{B}}^{T}
$$

subject to $\mathbf{Q}(0)=\mathbf{Q}_{\mathbf{0}}$. The output covariance, defined as $\mathbf{Y}(t)=\mathrm{E}\left[\tilde{\mathbf{y}}(t) \tilde{\mathbf{y}}^{T}(t)\right]$, reaches the 
steady-state Root Mean Square (RMS) value

$$
\tilde{\mathbf{y}}_{R M S}=\lim _{t \rightarrow \infty}\left(\operatorname{diag}\left[\tilde{\mathbf{C}} \mathbf{Q}(t) \tilde{\mathbf{C}}^{T}\right]\right)^{1 / 2}
$$

Notice that uncertainty in $\mathbf{p}$ makes $\tilde{\mathbf{y}}_{R M S}$ a random vector. If $y_{R M S}$ is a component of $\tilde{\mathbf{y}}_{R M S}$, a reliability metric that penalizes the violation $y_{R M S}>\bar{y}_{R M S}$ is given by $\bar{r}_{y R M S}\left(\bar{y}_{R M S}\right)$.

\section{Frequency-Domain}

The propagation of $f_{\mathbf{p}}(\mathbf{p})$ to the frequency domain leads to random processes of the form $x(\omega)$, fully prescribed by $F_{x(\omega)}(x, \omega)$. Here, $x(\omega)$ is any real frequency dependent metric of the feedback loop, e.g. Bode magnitude. This random process is parameterized by the frequency $\omega$ and the design variable $\mathbf{k}$. A reliability metric for $x(\omega)$ is $r_{x(\omega)}(\bar{x}(\omega), \underline{x}(\omega))$. For instance, conventional control requirements ${ }^{14}$ for disturbance rejection, noise attenuation and reference tracking can be cast in terms of the loop transfer function. In terms of the loop gain, namely $q(\omega)=|G K|, \underline{r}_{q(\omega)}(1)$ is a metric for low frequency requirements while $\bar{r}_{q(\omega)}(\bar{q}(\omega))$ with $\bar{q}(\omega)$ having a proper roll off, is the metric for high frequency requirements.

\section{Numerical Estimation}

In general, reliability metrics cannot be evaluated exactly since they involve the evaluation of complicated integrals, usually multi-dimensional, over complex domains. In this paper, reliability metrics are estimated using a hybrid approach which combines sampling and asymptotic approximations. Such an approach is based on the estimation of failure probabilities for the random variable. The estimation of failure probabilities for $x$ via sampling is given by

$$
P_{f} \approx \sum_{i=1}^{n} \frac{\mathcal{I}\left(x_{i} \in \mathcal{F}\right)}{n}
$$

where $\mathcal{I}(\cdot)$ is a binary indicator function that gives one if its argument is true and zero otherwise. The subscript in the above expression refers to samples of the dependent random variable $x$. An equivalent expression, where the limit state function is evaluated at the sample values of $\mathbf{p}$, can also be used. Usually, Monte-Carlo-Sampling (MCS $)^{4-6,15}$ is used to generate the required samples. 


\section{A. Hammersley-Sequence-Sampling (HSS)}

HSS generates representative deterministic samples of $f_{\mathbf{p}}(\mathbf{p})$. The error of approximating an integral by a finite sample of the integrand, e.g. Equation (15) instead of Equation (5), depends on the uniformity of the points used to generate the samples rather than on their randomness. This has motivated the development of deterministic sampling techniques such as HSS, where the distribution of points is optimized. The $n$ Hammersley samples are generated by transforming the $n$ Hammersley points $\mathbf{m}_{i}$ with $i=1,2, \ldots n$ through the inverse CDF of the uncertain parameter

$$
\mathbf{p}_{i}=F_{\mathbf{p}}^{-1}\left(\mathbf{m}_{i}\right)
$$

The Hammersley points can be generated ${ }^{16}$ easily. HSS requires far fewer samples ${ }^{17}$ than conventional MCS for a given confidence level. Improvements in the convergence rate of the estimated first two order moments by a factor of three to one hundred ${ }^{18}$ have been reported. In addition, if HSS is used to generate the samples for Equation (15) the estimated value of the failure probability is deterministic. In contrast, MCS leads to a random value for $P_{f}$ unless an infinite number of samples is used. This is especially noticeable if $n$ is small. The random character of the estimation can only be mitigated by increasing the number of samples, which incidentally increases the computational demands of algorithms based on MCS. Therefore, HSS not only leads to more accurate estimations than MCS for a given number of samples but also eliminates the random character of the results.

Figure 3 shows a comparison between HSS and MCS. In the top, $n=200$ points on the unit hypercube are shown. In the bottom, the corresponding samples for $f_{\mathbf{p}}(\mathbf{p})=f_{a}(a) f_{b}(b)$, where $f_{a}(a)=N(0,1)$ and $f_{b}(b)=B(3,2)$ with $\Delta_{b}=[0,1]$ are displayed. Here, $N$ and $B$ denote a Gaussian and a Beta distribution. Substantial differences in the uniformity of the points and in the clustering of the samples are observed.

\section{B. First-Order-Reliability-Method (FORM)}

FORM $^{11}$ uses an asymptotic approximation for the estimation of failure probabilities. In the process, $\mathbf{p}$ is transformed into the standard normal uncorrelated space $\mathbf{q}$. If $\mathbf{p}=\mathbf{T}(\mathbf{q})=$ 

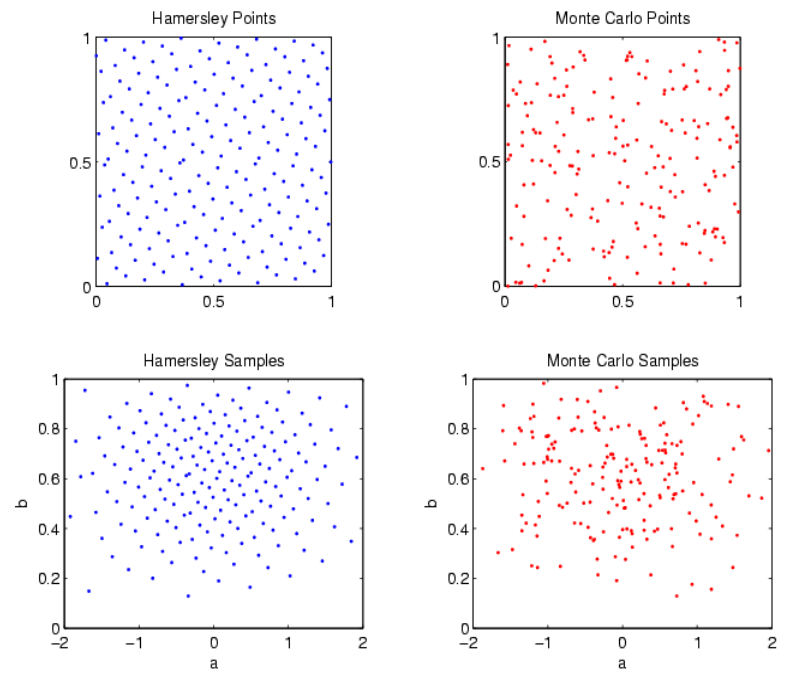

Figure 3. Points and samples via MCS and HSS.

$F_{\mathbf{p}}^{-1}\left(F_{\mathbf{q}}(\mathbf{q})\right)$, Equation (5) is equivalent to

$$
P_{f}=\int_{g(\mathbf{T}(\mathbf{q})) \leq 0} f_{\mathbf{q}}(\mathbf{q}) d \mathbf{q}
$$

FORM approximates the domain $g(\mathbf{T}(\mathbf{q})) \leq 0$, by a half-space fitted to the true domain at the point of maximum probability density. This approximation leads to

$$
P_{f} \approx \Phi\left(-\left\|\mathbf{q}^{*}\right\|\right)
$$

where $\mathbf{q}^{*}$, called the Most Probable Point (MPP), is given by the solution to the constrained optimization problem $\mathbf{q}^{*}=\operatorname{argi}\|\mathbf{q}\|, g\left(\mathbf{T}\left(\mathbf{q}^{*}\right)\right)=0$. In this expression, $\Phi$ refers to the CDF of a standard normal random variable. Notice that the rotational symmetry of $f_{\mathbf{q}}(\mathbf{q})$ leads to the one-dimensional approximation in Equation (17). The MPP does not exist when the probability of failure is zero or one since the equality constraint $g\left(\mathbf{T}\left(\mathbf{q}^{*}\right)\right)=0$ cannot be satisfied. Even though FORM is extensively used in structural engineering, its application to controls has been limited to stability ${ }^{10}$ analysis. The use of FORM to estimate failure probabilities related to $\lambda$ might lead to non-smooth limit state functions. This occurs when crossings between any pair of limit state functions corresponding to the system poles take place. 


\section{Hybrid Approach}

Sampling based techniques can readily be used to estimate probabilities of failure using Equation (15). However, high computational demands in the evaluation of $x_{i}=x\left(\mathbf{p}_{i}\right)$ can preclude their practicality especially when $P_{f} \approx 0$. Examples of this can be easily found ${ }^{\mathrm{c}}$. On the other hand, methods based on asymptotic approximations, such as FORM, provide good approximations when $P_{f}$ is small. This is clear since for failure probabilities away from zero and one, the slow decrease in $F_{\mathbf{q}}(\mathbf{q})$ near the MPP and the geometrical difference between the true limit state function and its linear approximation contribute a bigger error to the FORM approximation.

In this paper, a hybrid approach which combines HSS and FORM is used to estimate probabilities of failure. In order to identify the numerical tool that best suits the task at hand, a coarse and computationally-efficient estimation of $P_{f}$ is first generated using HSS. Such estimation is then compared with a reference, namely the reference failure probability $\rho$, a user-defined value set in advance. The comparison between the coarse estimate and $\rho$ is used to determine if either FORM or HSS are used to generate the more accurate estimation. Assume that two sets of Hammersley samples of $f_{\mathbf{p}}(\mathbf{p})$ are available. One set has $n_{1}$ samples and the other one has $n_{2}$ samples, where $n_{2} \gg n_{1}$. For a given failure domain $\mathcal{F}$ and a given reference failure probability $\rho$, proceed as follows

1. Estimate the $P_{f}$ using Equation(15) and the set of $n_{1}$ samples.

2. Recalculate $P_{f}$ as follows. If the estimated value is greater than $\rho$ use Equation (15) with the set of $n_{2}$ samples. If the estimated value is less than $\rho$ use FORM.

The refinement performed in Step 2 might not always be necessary. Situations in which this is the case are provided next. Since reliability metrics for random processes are heavily dependent on the larger values of the probabilities of failure that compose them, (see the bottom plot of Figure 2), refining the estimation of the relatively small values is inconsequential. Furthermore, if the reliability metrics are used to calculate the cost function of an optimization problem, more accurate estimations are not needed when the assessment resulting from using the coarse estimate of Step 1 denotes a poor control design, e.g. $\bar{r}_{\lambda}(0) \gg 0$.

\footnotetext{
${ }^{\mathrm{c}}$ Wang et al. ${ }^{5,15}$ make the approach computationally viable by using a single random variable to model 28 uncertain parameters. Same authors ${ }^{6}$ require 25000 samples to determine a sufficiently small $95 \%$ confidence interval.
} 
The above procedure applies to the random variable $x$. Extensions to random processes are easily attained. For the random process $x(h)$, generate $e$ samples in the $h$ domain as follows

$$
h_{i}=\frac{i-1}{e-1}\left(h_{\max }-h_{\min }\right)+h_{\min }, i=1,2 \ldots e
$$

where $h_{\max }$ and $h_{\min }$ are the bounds of the interval of interest, e.g. $h_{\max }=h_{2}$ and $h_{\min }=h_{1}$ in Equation (10). Reliability metrics for the $e$ random variables $x\left(h_{i}\right)$, are estimated via the hybrid approach and then used to form the integrands in Equations (10-11). For the sake of clarity, we will refer to the $e$ samples in the $h$ domain as the $e$ partitions.

\section{Control Synthesis}

\section{A. Problem Formulation}

The formulation of the control design problem from a reliability perspective is as follows. For a given set of design requirements, plant model, compensator structure and uncertainty model we would like to find the compensator parameters for which the resulting probability of violating the controlled system requirements is minimized. Such requirements combine stability and performance specifications in time and frequency domains. Notice that this statement refers to the excursion of the outcomes into the failure domains. Performance improvements regarding the outcomes within the admissible domains will also be considered. Such improvements are attained by dynamically shaping the failure domains during the minimization of the reliability cost metrics. This topic is considered next.

\section{B. Performance Improvements}

The reliability metrics in Equations (6-9) are applied using a fixed failure set $\mathcal{F}$. In this form, a reliability analysis cannot assess the system's performance in the regions where the design requirements are satisfied, i.e. the union of the admissible domains $\mathcal{A}$ associated with all the design requirements. Since the portion of the random outcome lying on the admissible domain $\mathcal{A}$ might end up being substantially larger than the portion lying on the failure domain $\mathcal{F}$, a reliability-based approach with fixed failure envelopes do not have control over the bulk portion of the PDF, which is the portion that dictates the most likely overall performance. 
The ideas behind the approach to be proposed will be introduced with an example. Let $x(\mathbf{k})$ be the stationary RMS value of an error signal. Usually, we would like to find $\mathbf{k}$ such that $x$ is as close as possible to zero. Uncertainty in the plant makes $x$ a random variable. Let $\bar{x}$ be the failure envelope associated with a design requirement, i.e. $\mathcal{F}=\{x \mid x \in[\bar{x}, \infty)\}$. The minimization of $\bar{r}_{x}(\bar{x})$ leads to a reliability optimal compensator. Suppose there exist multiple designs leading to $\bar{r}_{x}(\bar{x})=0$. These designs however differ in how well the resulting PDF of $x$ spreads over the admissible domain $\mathcal{A}=\{x \mid x \in[0, \bar{x})\}$. The concentration of $f_{x}(x)$ about zero is an indicator of the overall performance. Say, $\mathbf{k}_{1}$ leads to $\bar{r}_{x}(\bar{x} / 2)=0$ and $\mathbf{k}_{2}$ leads to $\bar{r}_{x}(\bar{x})=0$. Since none of these two designs violate the design requirement $x>\bar{x}$, a reliability analysis cannot establish that the compensator with parameters $\mathbf{k}_{1}$ has a better global performance than the one which uses $\mathbf{k}_{2}$.

By minimizing the reliability metrics and simultaneously enlarging the failure domain(s), the whole random variable/process can be concentrated about regions where an improved system performance is achieved. This is attained by parameterizing both the failure envelopes and a failure size penalizing function with an additional design variable. This variable will be denoted as c. For the RMS example above, the minimization of $J=\bar{r}_{x}(c)+c$, where the design variable is $\mathbf{d}=[\mathbf{k}, c]$ and $c \in[0, \bar{x}]$, leads to solutions which integrate reliability and performance considerations on a single formulation. Notice that the value of $J$ for $\mathbf{k}_{1}$ is less than the one for $\mathbf{k}_{2}$ if $c \in[\bar{x} / 2, \bar{x})$.

In general, we will refer to the augmented reliability metric as the sum of a reliability metric from Section III and a penalizing term. Augmented reliability metrics for the random variable $x$ and the random process $x(h)$ take the form $r_{x}(\underline{x}(\mathbf{c}), \bar{x}(\mathbf{c}))+\gamma_{x}(\mathbf{c})$ and $r_{x(h)}(\underline{x}(h, \mathbf{c}), \bar{x}(h, \mathbf{c}))+\gamma_{x(h)}(\mathbf{c})$ respectively. The penalizing functions $\gamma_{x}(\mathbf{c})$ and $\gamma_{x(h)}(\mathbf{c})$ must be proportional to the size of the admissible domain $\mathcal{A}$. In addition, they must be built such that the minimization of the augmented metric does not lead to inadmissible solutions, e.g. $r_{x}=1$ and $\gamma_{x}=0$. If $r<\epsilon$ is desired, use a monotonically increasing function satisfying $\gamma \in[0, \epsilon]$. For the RMS example above, the minimization of the augmented metric leads to $\bar{r}_{x}<\epsilon$ if $\gamma_{x}(c)=\epsilon c / \bar{x}$ for $c \in[0, \bar{x}]$.

\section{Synthesis Procedure}

A step-by-step procedure to reliability-based control synthesis is presented next.

1. Determine the plant model and the control structure. First principles and classical deterministic approaches to compensator design can be used. Identify the set of pa- 
rameters that have a strong impact on the plant model. Use sensitivity information and engineering judgment to select the set of uncertain parameters $\mathbf{p}$. At this stage, the parametric plant model, e.g. $G(\mathbf{p})$, and the control structure, e.g. $K(\mathbf{k})$, must be fully determined.

2. Generate the probabilistic parameter model $f_{\mathbf{p}}(\mathbf{p})$. Use engineering judgment and experimental data if available.

3. Determine the number of HSS samples $n_{1}$ and $n_{2}$ to be used. Follow the guidelines provided in Section IV-C. Use Equation (16) to generate the sample sets of $f_{\mathbf{p}}(\mathbf{p})$ for both $n_{1}$ and $n_{2}$.

4. Cast the violation of the design requirements in terms of reliability metrics as in Equations (6-9). Recall that specific realizations for stability, time and frequency requirements were provided in Section III-C. Use these metrics to compose the reliability cost vector $\mathbf{r}$. This step requires determining the failure domain $\mathcal{F}$ corresponding to each component of $\mathbf{r}$.

5. Determine which failure domains will remain fixed during synthesis and which ones will be dynamically shaped. Let $\mathbf{d}$ be the design variable. When the failure domains are fixed $\mathbf{d}=\mathbf{k}$. When failure domains are shapeable $\mathbf{d}=\left[\mathbf{k}^{T}, \mathbf{c}^{T}\right]^{T}$.

6. Build a penalizing function $\gamma(\mathbf{c})$ for each of the components of $\mathbf{r}$ whose failure domain is not fixed. Follow the guidelines in Section V-B. Update the components of the reliability cost vector $\mathbf{r}$ by adding the penalizing functions and parameterizing the failure envelopes.

7. Solve the multi-objective optimization problem

$$
J=\min _{\mathbf{d}}\left\{\mathbf{r}^{T} \mathbf{N w}\right\}
$$

where $\mathbf{N}$ is a diagonal normalization matrix such that the components of $\mathbf{r}^{T} \mathbf{N}$ are between zero and one, and $\mathbf{w}$ is composed of non-negative weights. The matrix $\mathbf{N}$ is used to facilitate the weighting of the components of $\mathbf{r}$.

Each cost function evaluation used in the search for the optimal reliability-based design $\mathbf{d}^{*}$ requires a reliability analysis. This analysis is done by calculating the reliability 
metrics contained in $\mathbf{r}$ using the hybrid approach. This task requires forming the closed-loop Equations (4-14) and performing typical control studies such as finding closed loop poles, time responses and Bode plots.

During optimization, the following procedure is suggested in order to focus most of the computational effort toward the assessment of better designs. First, calculate the cost function using $n_{1}$ samples for $e_{1}$ partitions. This implies that only the first step of the hybrid approach is applied to all reliability metrics. This first assessment, denoted as $A_{1}$, should be computationally efficient. If $A_{1}$ shows that $\mathbf{d}$ is a good design relative to the ones already evaluated by the optimizer, perform the refined assessment $A_{2}$. The assessment $A_{2}$ is carried out by using $e_{2}$ partitions and a adjustable value for the reference failure probability $\rho$. The adjustment of $\rho$ is done to prevent inconsequential calculations as it was mentioned in Section IV-C. If the particular failure probability is to be estimated via HSS use $n_{2}$ samples and Equation (15). If the particular failure probability is to be estimated via FORM use Equation (17). If $A_{1}$ indicates that $\mathbf{d}$ is not a good design, $A_{2}$ is not carried out. This two-fold analysis is applied to all designs the optimizer evaluates in the search for $\mathbf{d}^{*}$.

Implementing the dual assessment described above avoids the inconsequential refinement of the reliability metrics.

\section{Optimization and Reliability}

Due to the nature of the reliability metrics in $\mathbf{r}$, the cost function $J(\mathbf{p}, \mathbf{d})$ might not only have plateaus, i.e., there could exist a design $\mathbf{d}$ and a non-zero perturbation $\delta$ such that $J(\mathbf{p}, \mathbf{d})=J(\mathbf{p}, \mathbf{d}+\delta)$, but might also have a discontinuous gradient.

The use of sampling in the estimation of probabilities makes the cost function discontinuous at every point of the design space. Let $\hat{J}(\mathbf{p}, \mathbf{d})$ be an estimation of the actual cost $J(\mathbf{p}, \mathbf{d})$. For any design $\mathbf{d}$ and regardless of the number of samples, there always exists a perturbation $\delta$ such that $\hat{J}(\mathbf{p}, \mathbf{d})=\hat{J}(\mathbf{p}, \mathbf{d}+\delta)$. This situation is aggravated, i.e. bigger perturbations can be found, when a smaller number of samples is used or when $P_{f}$ is close to zero or one.

The discontinuous nature of the estimated value of $J$ must be taken into account when selecting a numerical optimization method to solve Equation (18). In the examples to follow, the resulting non-convex non-continuous optimization problem is first solved using Genetic- 
Algorithms (GA) for a fixed number of generations. Since GA is based on a random search, the twofold procedure described above is particularly convenient. After the fixed number of generations is reached the GA solution is refined using the Nelder Mead Simplex algorithm, which is a local non-gradient based search method.

\section{Numerical Examples}

The synthesis procedure of Section V-C is applied herein. A textbook satellite attitude control problem is considered first. Then, disturbance rejection for a flexible beam is presented. If $\mathbf{p} \in \mathbb{R}^{m}$, the parameters used for $A_{1}$ are $n_{1}=75 \mathrm{~m}$ and $e_{1}=90$. For $A_{2}$ we use $n_{2}=500 m$ and $e_{2}=180$. For the sake of comparison, the examples present the solution to deterministic versions of the problems for which the expected value of $\mathbf{p}$ is used. Such problems and the corresponding solutions are referred to as the nominal ones.

\section{A. Attitude Control}

Accurate satellite pointing in the presence of large thermal gradients and mass losses for uncertain initial conditions is desired. A simple rotational model of two bodies connected with a flexible boom leads to

$$
\begin{aligned}
& J_{1} \ddot{\theta}_{1}+b\left(\dot{\theta}_{1}-\dot{\theta}_{2}\right)+k\left(\theta_{1}-\theta_{2}\right)=u \\
& J_{2} \ddot{\theta}_{2}+b\left(\dot{\theta}_{2}-\dot{\theta}_{1}\right)+k\left(\theta_{2}-\theta_{1}\right)=0
\end{aligned}
$$

where $\theta_{1}$ and $\theta_{2}$ are the deflection angles, $J_{1}$ and $J_{2}$ are moments of inertia, $k$ is the equivalent stiffness, $b=a \sqrt{k / 10}$ is the equivalent damping coefficient and $u$ is the applied torque. The variable $a$ is used to model the changes in damping caused by thermal variations. We assume that $J_{2}=0.1$ since mass losses only affect $J_{1}$. The non-collocated sensor-actuator pair resulting from using $y=\theta_{2}$ leads to the SISO transfer function for the plant

$$
G(\mathbf{p})=\frac{k+b s}{J_{1} J_{2} s^{4}+b\left(J_{1}+J_{2}\right) s^{3}+\left(J_{1}+J_{2}\right) k s^{2}}
$$

Variations in the operating conditions and the ignorance on the initial conditions are modeled using $\mathbf{p}=\left[J_{1}, e, k, \theta, \dot{\theta}\right]^{T}$, where $\theta=\theta_{1}(0)=\theta_{2}(0)$. The following output-feedback control 
structure is assumed

$$
K(\mathbf{k})=\frac{k_{1}+k_{2} s+k_{3} s^{2}+k_{4} s^{3}}{k_{5}+k_{6} s+k_{7} s^{2}+k_{8} s^{3}}
$$

The joint PDF that describes the uncertainty in $\mathbf{p}$ is given by the independent random variables listed in Table 1, where $U$ and $B$ refer to Uniform and Beta distributions. Notice that the Beta distribution has four independent parameters, two of them are the conventional arguments and the other two are the support bounds. Performance requirements

Table 1. Uncertainty model.

\begin{tabular}{|c|c|c|}
\hline$J_{1}$ & $\Delta_{J 1}=[0.8,1]$ & $f_{J 1}\left(J_{1}\right)=U(0.8,1)$ \\
\hline$a$ & $\Delta_{a}=[0.03,0.2]$ & $f_{a}(a)=B(0.3,0.2)$ \\
\hline$k$ & $\Delta_{k}=[0.09,0.4]$ & $f_{k}(k)=B(5,5)$ \\
\hline$\theta$ & $\Delta_{\theta}=[-\pi / 2, \pi / 2]$ & $f_{\theta}(\theta)=B(5.2,5.2)$ \\
\hline$\dot{\theta}$ & $\Delta_{\dot{\theta}}=(-15,15)$ & $f_{\dot{\theta}}(\dot{\theta})=B(2.5,2.5)$ \\
\hline
\end{tabular}

on the system's closed-loop stability, settling time, over-shoot, and control usage for a step response and on the magnitude of the loop transfer function lead to $\mathbf{r}=\left[\bar{r}_{\lambda}(\bar{\lambda})\right.$, $\left.r_{y(t)}(\underline{y}(t), \bar{y}(t)), r_{u(t)}(\underline{u}, \bar{u}), \underline{r}_{q(\omega)}(\underline{q}(\omega)), \bar{r}_{q(\omega)}(\bar{q}(\omega))\right]^{T}$, where $q(\omega)=|G K|$ is the loop gain. The failure envelopes to be used are $\bar{\lambda}=0 ; y(t)=-1.25 \mathcal{H}(t)+2.2 \mathcal{H}(t-70)$ for $t \in[0,80]$, where $\mathcal{H}$ is the Heaviside function; $\bar{y}(t)=1.25 \mathcal{H}(t)-0.2 \mathcal{H}(t-70)$ for $t \in[0,80] ; \underline{u}(t)=-0.5$ for $t \in[0,25] ; \bar{u}(t)=0.5$ for $t \in[0,25] ; \underline{q}(\omega)=0.75 / \omega$ for $\omega \in\left[10^{-6}, 0.2\right]$ and $\bar{q}(\omega)=1$ for $\omega \in\left[1,10^{2}\right]$. These failure domains lead to the normalization matrix $N=\operatorname{diag}\{[1,80,25,0.2-$ $\left.\left.10^{-6}, 10^{2}-1\right]\right\}$. Note that overshoot and settling time constrains are integrated using $y(t)$. Fixed failure domains are assumed. This leads to $\mathbf{d}=\left[k_{1}, k_{2}, k_{3}, k_{4}, k_{5}, k_{6}, k_{7}, k_{8}\right]^{T}$. The solution of the optimization problem in Equation (18) requires calculating $\mathbf{r}$ for multiple control designs. Recall that for each design, the hybrid approach of Section $\mathrm{V}-\mathrm{C}$ is used to generate the coarse assessment $A_{1}$ and eventually the refined assessment $A_{2}$. For $A_{1}$, use $n_{1}=375$ samples, $e_{1}=90$ partitions and Equation (15). If $A_{2}$ is required, use $e_{2}=180$ partitions, the reference failure probability $\rho=0.01$ and the results of $A_{1}$. For the failure probabilities to be estimated via HSS use $n_{2}=2500$ samples. In this example, FORM is used to calculate $\bar{r}_{\lambda}(0)$ for $\rho=0.01$. Before presenting the results from the above procedure, the deterministic problem based on the expected value of the parameter is considered. 


\section{Nominal Compensator}

A baseline compensator for the nominal plant is designed by standard pole placement techniques such that large stability margins are attained. This practice results in the nominal compensator parameters $\mathbf{k}=10^{6}[0.0108,-0.3271,0.1192,0.0092,1.8835,2.1305,2.2276$, $0.9308]^{T}$. A reliability analysis of the nominal compensator using the probabilistic uncertainty prescribed by $f_{\mathbf{p}}(\mathbf{p})$ leads to $\mathbf{r}^{T} \mathbf{N}=\left[0,0.2485,0.227,7.08 \times 10^{-5}, 0\right]$. This vector indicates that the closed-loop system is robustly stable, i.e. $\bar{r}_{\lambda}(0)=0$, but the time responses are unsatisfactory. The CDF of $\lambda$ as well as the time evolutions of the output and the control signals are shown in Figures 4-6. The sudden variation in the slope of the CDF of Figure 4 is the result of a change in the closed-loop pole that determines $\lambda$. The considerable disparity between $\lambda(E[\mathbf{p}])$ and $E[\lambda(\mathbf{p})]$ shows that the nominal problem is not a meaningful representative of the probabilistic behavior. Figures 5 and 6 show the time evolution of the random signals by indicating the 1, 10, 20, 30, 40, 50, 60, 70, 80, 90 and 99 percentiles. In Figures 5 and 6, the percentiles, the nominal fields and the failure envelopes are shown. Dotted lines are used to indicate the the failure envelopes. It is interesting to see how the PDFs expand, e.g. Figure 6 at 2.5 and 8 seconds, and contract, e.g. Figure 6 at 4 and 16 seconds, in a oscillatory manner. This information can be used to determine the time periods when the effects of uncertainty are more noticeable.

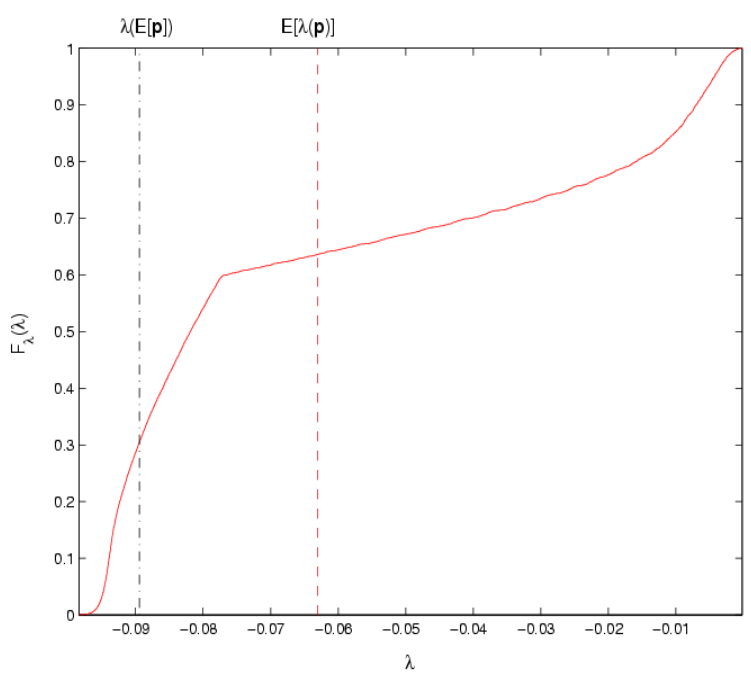

Figure 4. $\lambda$ for the nominal compensator. 

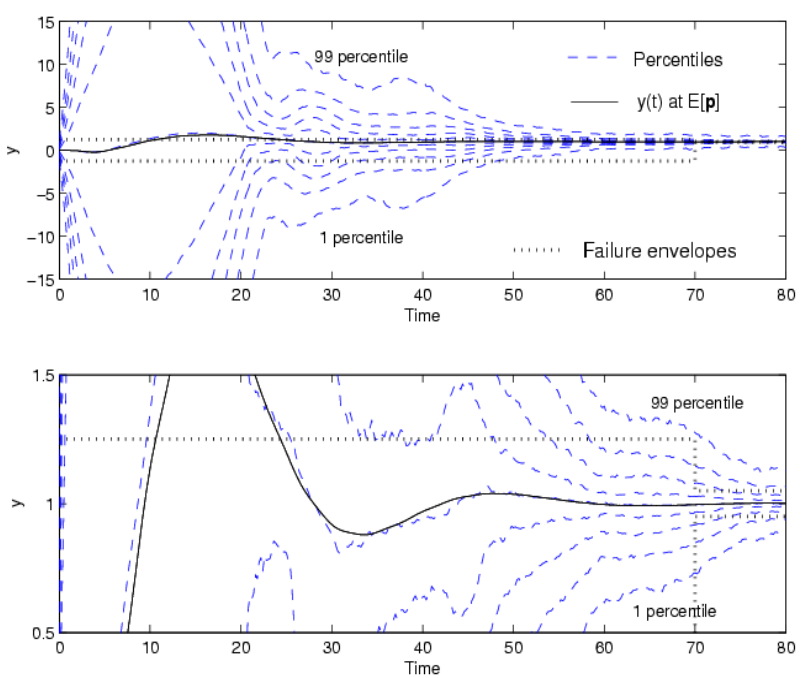

Figure 5. $y(t)$ for the nominal compensator. A zoom is shown below.

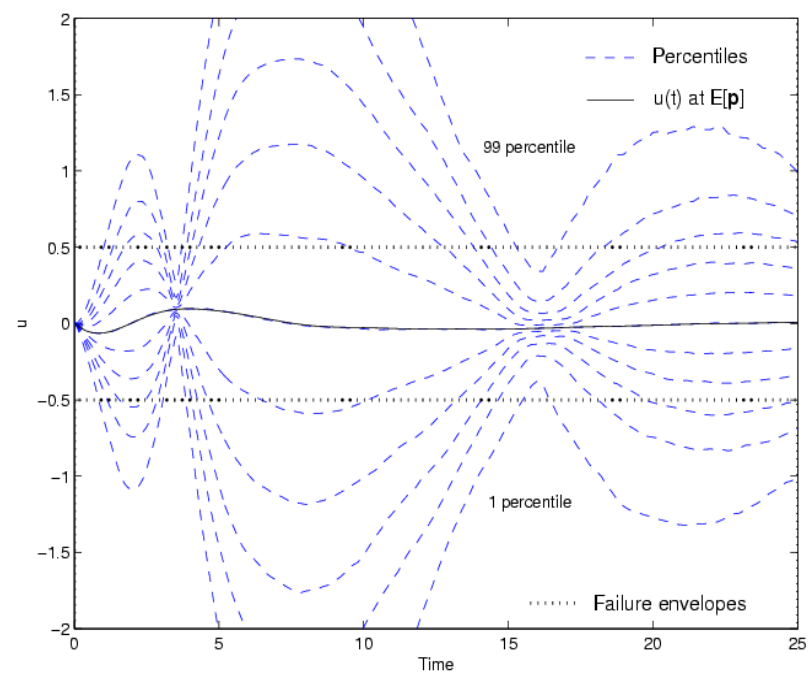

Figure 6. $u(t)$ for the nominal compensator.

\section{Reliability-based compensator}

The synthesis procedure listed above leads to $\mathbf{d}^{*}=10^{6}[0.0405,0.1267,0.2422,0.0320,0.5244$, $1.0057,1.2263,0.6560]^{T}$ and $\mathbf{r}^{T} \mathbf{N}=\left[3.13 \times 10^{-4}, 0.0521,0.0918,1.33 \times 10^{-4}, 0\right]$ for which the weighting vector $\mathbf{w}=[500,1,1,1,1]^{T}$ was used. A probabilistic analysis of this compensator 
leads to Figures 7-10. Notice that despite of the increased variability of the dominant closedloop poles resulting from this compensator $\left(\Delta_{\lambda}\right.$ in Figure 7 is about three times larger than the one shown in Figure 4), stability is compromised with only $3.13 \times 10^{-4}$ probability. Notice that a substantial improvement in the performance is achieved by trading-off a very small margin of the probability of instability. This improvement can be seen after comparing Figures 5-6 with 8-9. Better robust stability characteristics could be attained by increasing the weight in $\mathbf{w}$ for the corresponding component of $\mathbf{r}$. Recall that reaching zero probability of instability might be unfeasible. From Figure 9 we see that for all possible parameter values and initial conditions the process $u(t)$ stays between the \pm 0.5 range with more than 0.8 probability after 6 seconds. Figure 10 shows that uncertainty mostly affects the damping and the location of the resonant frequency. Violations of the low frequency requirement are completely avoided. Overall, the performance resulting from $\mathbf{d}^{*}$ is substantially better than the one resulting from $\mathbf{k}$.

During optimization, 157 random variables were used to evaluate $\mathbf{c}$ for the coarse assessment $\mathcal{A}_{1}$. Such task takes 23.6 seconds when performed on a Pentium III $1795 \mathrm{MHz}$ with $512 \mathrm{MB}$ of RAM. Notice that the CPU time associated with $\mathcal{A}_{2}$ depends on the initial conditions used to find the MPPs. For this assessment, HSS was used for 628 random variables and the hybrid approach was used for robust stability. This task took 102 seconds.

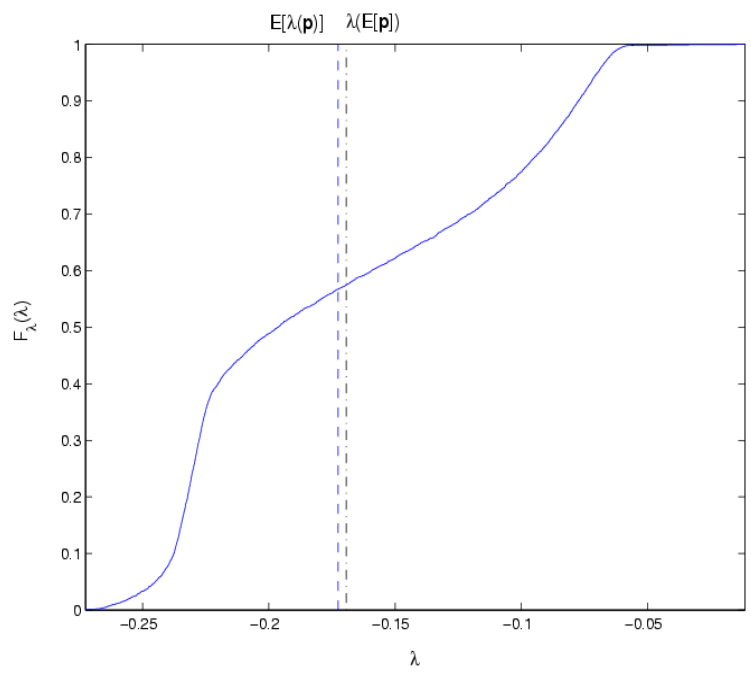

Figure 7. $\lambda$ for the reliability-based optimal compensator. 

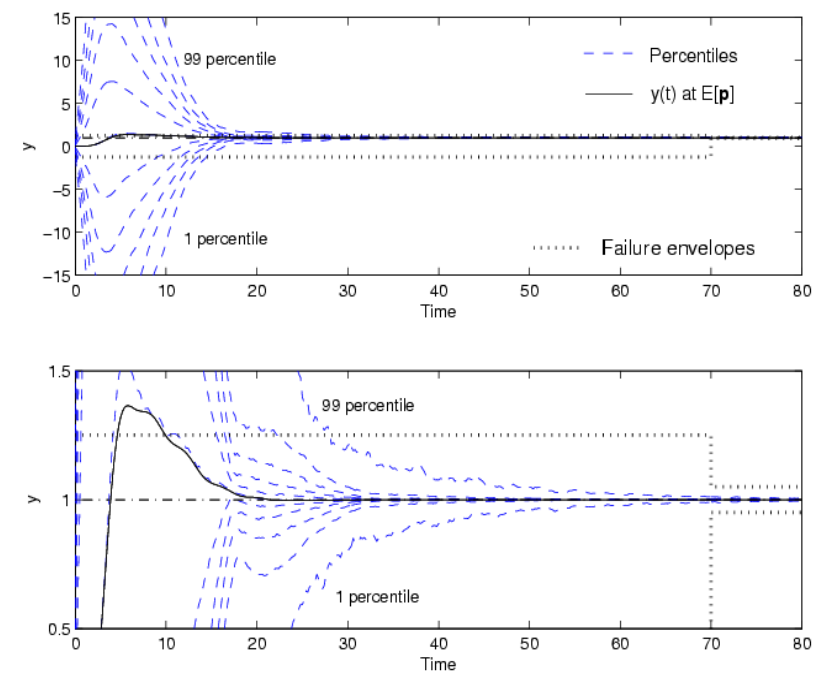

Figure 8. $y(t)$ for the reliability-based optimal compensator. A zoom is shown below.

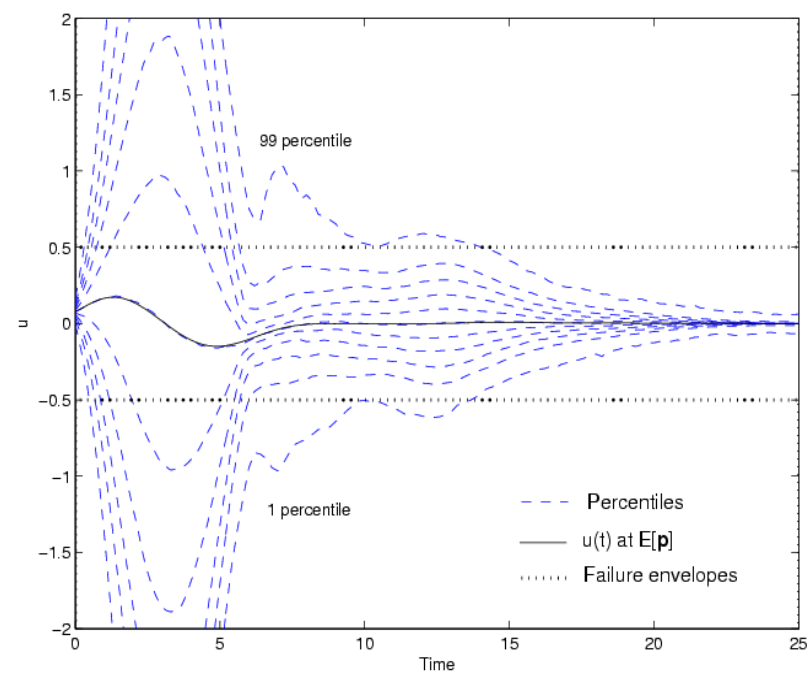

Figure 9. $u(t)$ for the reliability-based optimal compensator.

\section{B. Disturbance Rejection for a Flexible Beam}

The second example will focus on a reliability-based disturbance rejection solution for a flexible beam test article with both physical and modal parameter uncertainties. The system, displayed in Figure 11, consists of a very flexible thin aluminum blade, approximately one- 


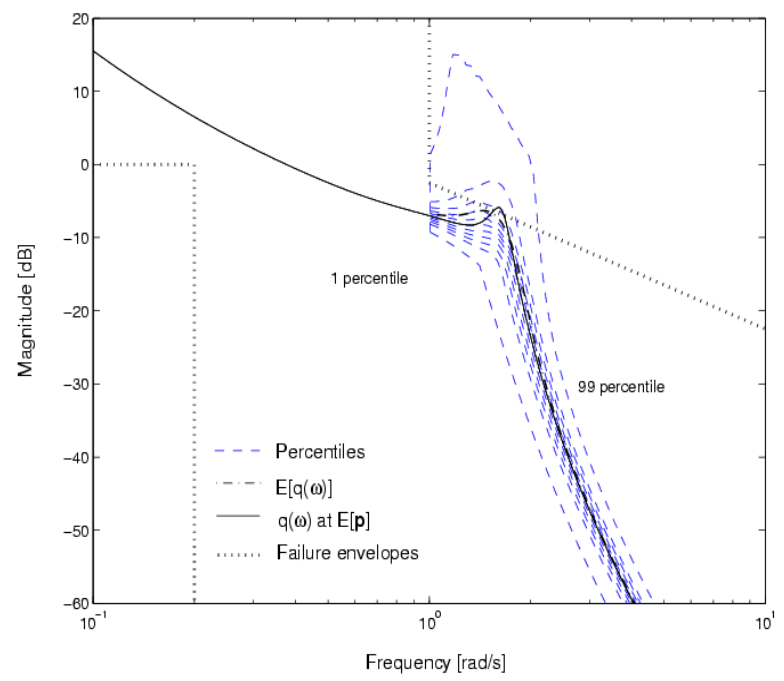

Figure 10. Bode plot of the loop gain for the reliability-based optimal compensator.

meter long, attached at its base to a hub motor. The hub motor is the control actuator for the system. At the tip of the beam, there is a reaction wheel that serves as a disturbance generator. The test article has nine sensors that may be used in any combination for either feedback or performance output monitoring. The finite element method is used to model this system by utilizing Euler-Bernoulli planar beam elements. A complete description of the flexible beam test article $^{19}$ is available.

For this paper we study a SISO model of the system in which the input $\mathbf{u}$ is the hub motor torque and the measured output $\mathbf{y}$ is the tip velocity. The tip reaction wheel disturbance is modeled by passing a Gaussian white noise process through a second-order linear lowpass filter, with parameters $\zeta=0.8$ and $\omega_{n}=200 \pi \mathrm{rad} / \mathrm{s}$. The first five modes of the elastic structure are used to build a state space realization of the plant. This, in addition to the disturbance model leads to a open-loop system where $\mathbf{x} \in \mathbb{R}^{12}, \mathbf{u} \in \mathbb{R}$ and $\mathbf{y} \in \mathbb{R}$. The uncertain parameters are the Young's Modulus $E(\mathrm{~Pa})$, the density $\rho\left(\mathrm{Kg} / \mathrm{m}^{3}\right)$ and the damping ratios of the retained vibration modes. This set leads to $\mathbf{p}=\left[E, \rho, \xi_{1}, \xi_{2}, \xi_{3}, \xi_{4}, \xi_{5}\right]^{T}$, whose components are assumed independent. The corresponding PDFs are given in Table 2. The mean value of the parameters $E[\mathbf{p}]$ is set to coincide with the parameters in the finite element model. These mean values were chosen to match experimental data, while the supports of the distributions were set according to reasonable ranges of variation. The shapes of the PDFs were arbitrarily set. Performance requirements on stability and the 


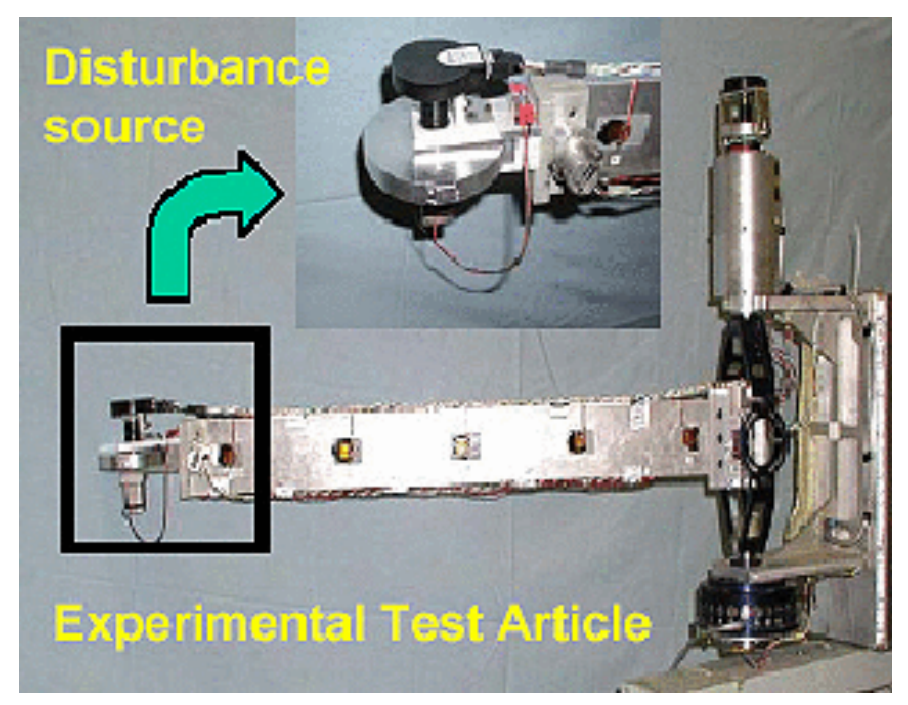

Figure 11. Flexible beam test article.

Table 2. Uncertainty model.

\begin{tabular}{|c|c|c|}
\hline$E$ & $\Delta_{E}=10^{10}[5.226,7.839]$ & $f_{E}(E)=B(5,5)$ \\
\hline$\rho$ & $\Delta_{\rho}=[2280,3420]$ & $f_{\rho}(\rho)=B(3,3)$ \\
\hline$\xi_{1}$ & $\Delta_{\xi_{1}}=[0.08,0.12]$ & $f_{\xi 1}\left(\xi_{1}\right)=B(2,2)$ \\
\hline$\xi_{2}$ & $\Delta_{\xi_{2}}=[0.0252,0.0378]$ & $f_{\xi 2}\left(\xi_{2}\right)=B(2,2)$ \\
\hline$\xi_{3}$ & $\Delta_{\xi_{3}}=[0.02,0.03]$ & $f_{\xi 3}\left(\xi_{3}\right)=B(2,2)$ \\
\hline$\xi_{4}$ & $\Delta_{\xi_{4}}=[0.0304,0.0456]$ & $f_{\xi 4}\left(\xi_{4}\right)=B(2,2)$ \\
\hline$\xi_{5}$ & $\Delta_{\xi_{3}}=[0.02,0.03]$ & $f_{\xi 5}\left(\xi_{5}\right)=B(2,2)$ \\
\hline
\end{tabular}

output RMS lead to $\mathbf{r}=\left[\bar{r}_{\lambda}(0), \bar{r}_{y R M S}\left(\bar{y}_{R M S}\right)\right]^{T}$. Two control design problems are considered in this example, output feedback and full-state feedback with a full-order observer.

\section{Output Feedback}

First, a third-order compensator with the same structure of Equation (20) is considered. A baseline compensator is designed such that the tip velocity RMS for the nominal plant is minimized. This results in $y_{R M S}=0.025 \mathrm{~m} / \mathrm{s}$ and $\mathbf{k}=[-0.0052,-0.2589,-16.1462,-5.007$, $0.0004,0.0009,0.2519,0.0598]^{T}$. A reliability analysis of the nominal compensator for the uncertainty model in Table 2 leads to $\bar{r}_{\lambda}(0)=0.068$ and $\bar{r}_{y R M S}(0.05)=0.010$. 
For the reliability based-design, a shapeable failure domain for the RMS component of $\mathbf{r}$ is assumed. This leads to the cost vector $\mathbf{r}=\left[\bar{r}_{\lambda}(0), \bar{r}_{y R M S}(c)+\gamma_{y R M S}\right]^{T}$, where $c \in[0,0.05]$ and $\gamma_{y R M S}=c$. The corresponding design variable is $\mathbf{d}=\left[\mathbf{k}^{T}, c\right]^{T}$ and the normalization matrix is $\mathbf{N}=\operatorname{diag}\{[1,1.05]\}$. The weighting vector $\mathbf{w}=[20,1]^{T}$ leads to $\mathbf{d}^{*}=$ $\left[-0.0072,-0.3506,-22.2811,0.0145,0.0006,0.0019,0.3761,2.8 \times 10^{-8}, 0.0305\right]^{T}, \bar{r}_{\lambda}(0)=0$ and $\bar{r}_{y R M S}(0.0305)=4.33 \times 10^{-3}$. A probabilistic analysis of $\mathbf{d}^{*}$ leads to Figures $12-13$. Figure 12 shows that the the whole random variable $y R M S$ is moved towards zero, by virtue of the non-fixed failure envelope. Figure 13 shows Bode magnitude plots of the disturbance to output transfer function, namely $T_{z y}$, Notice that differences in the low-frequency portion of the diagram have a bigger impact on the RMS value. In this Figure, Bode magnitude plots for the nominal compensator and the reliability optimal compensator are shown. The 1, 10, 20, 30, 40, 50, 60, 70, 80, 90 and 99 percentiles and the nominal fields are superimposed.

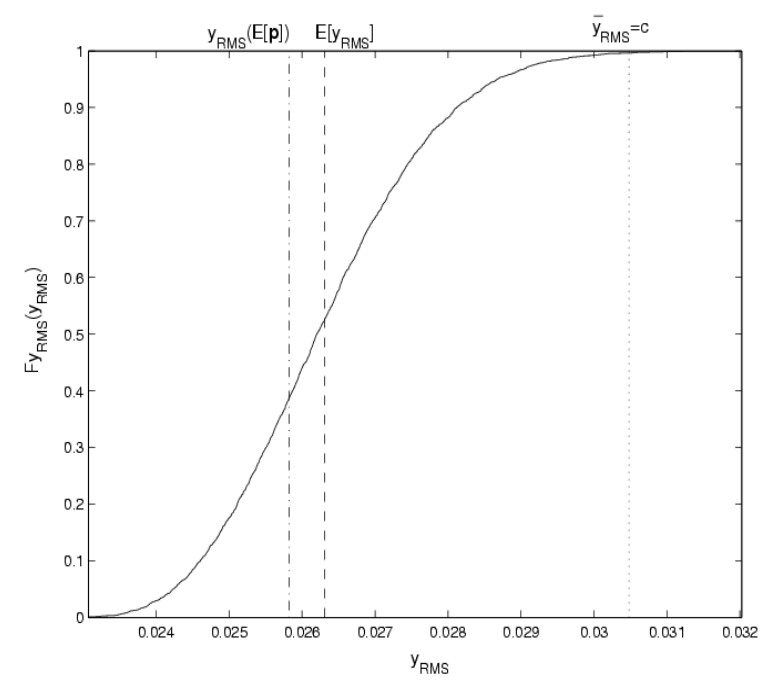

Figure 12. Tip velocity RMS for the output feedback solution.

\section{Full-State Feedback and Full-Order Estimation}

Second, full-state feedback and a full-order observer define the structure of the compensator is considered. Hence, the feedback gain $\mathbf{G}$, the observer gain $\mathbf{L}$ and the RMS failure envelope $c$ are the design variables. Recall that the separation principle does not hold due to uncertainty in the plant. The resulting closed-loop dynamics is given by Equations (3) and (4). Notice 


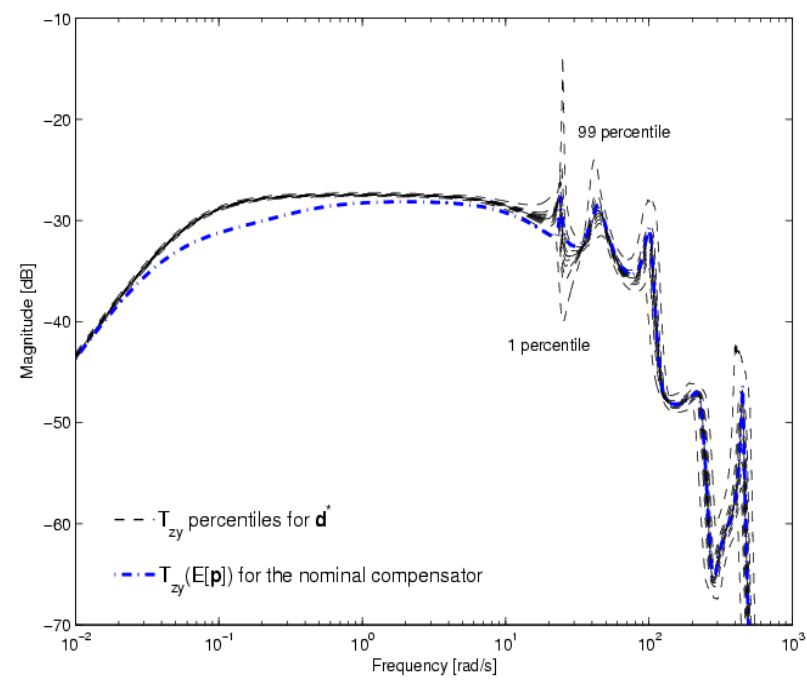

Figure 13. Bode diagrams of $T_{z y}$ for output feedback.

that although the observer is deterministic, all the closed-loop poles are random. As before, a baseline compensator for the nominal plant is first designed by minimizing the RMS value, which results in $y_{R M S}=0.011 \mathrm{~m} / \mathrm{s}$. Using $\bar{y}_{R M S}=0.05 \mathrm{~m} / \mathrm{s}$, this compensator leads to $\bar{r}_{\lambda}(0)=0.232$ and $\bar{r}_{y R M S}(0.05)=0.002$.

The reliability-based synthesis approach for the same setup used in the output feedback example leads to $\bar{r}_{\lambda}(0)=0$ and $\bar{r}_{y R M S}(0.0139)=3.6 \times 10^{-3}$. Due to the large number of elements in $\mathbf{d}^{*}$, only $c=0.0139 \mathrm{~m} / \mathrm{s}$ is provided. The probabilistic analysis of $\mathbf{d}^{*}$ leads to Figures 14-15. Compared with Figure 12, Figure 14 shows considerably more variability in the Bode magnitude plot as well as a significant reduction in the damping of the first mode. Since there is no conservatism in the selection of the nominal plant, i.e. $G(\mathrm{E}[\mathbf{p}])$ is not the most difficult plant to control, the optimal deterministic values for the nominal plant don't have to bound the resulting supports, e.g. values in $\Delta_{y R M S}$ may be less than $y_{R M S}=0.011$ $\mathrm{m} / \mathrm{s}$.

It is interesting to notice that even though $\mathbf{d}^{*}$ leads to a robustly stable closed-loop system in Equation (3), the full-state feedback subsystem $\tilde{\mathbf{A}}_{1,1}$ and the full-order observer subsystem $\tilde{\mathbf{A}}_{2,2}$ have a non-zero probability of instability. This indicates that the Separation Principle artificially reduces the design space. While robust stability was achieved in both output feedback and full-state feedback solutions, the latter led to a better performance. 


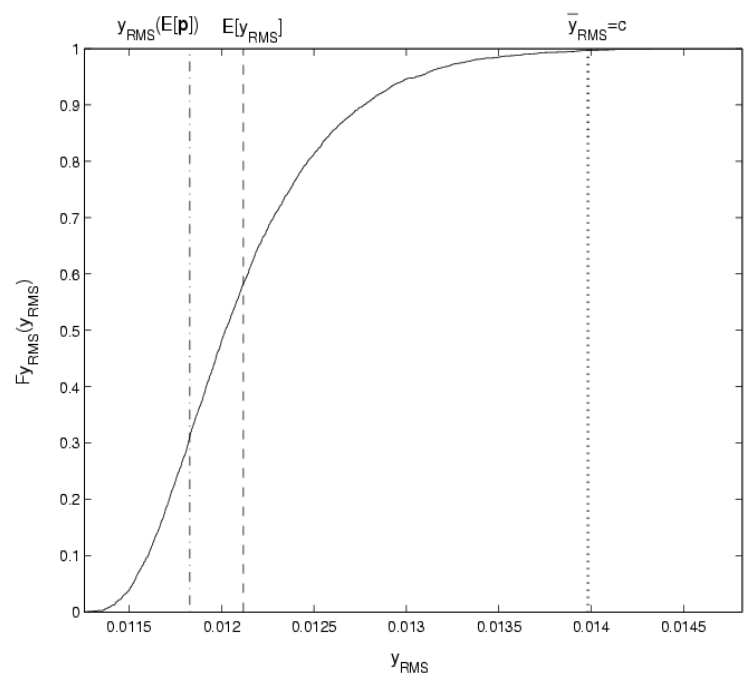

Figure 14. Tip velocity RMS for the full-state feedback solution.

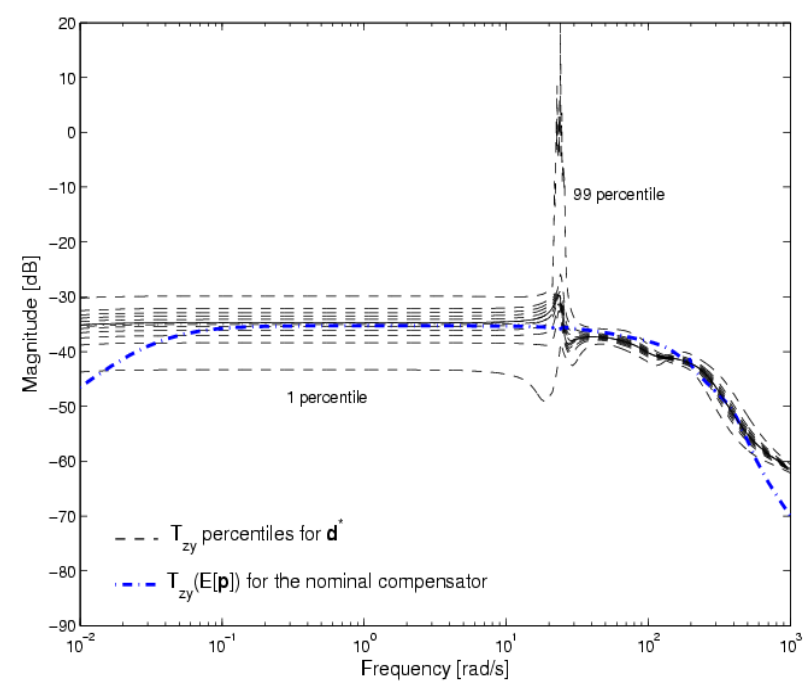

Figure 15. Bode diagrams of $T_{z y}$ for full-state feedback for full-order observer.

\section{Conclusions}

This paper proposes a reliability-based control synthesis method for systems with probabilistic uncertainty. Control synthesis is performed by solving a multi-objective optimization problem where the probability of violating stability and performance requirements is min- 
imized while the failure domains are simultaneously enlarged. Including dynamically shapeable failure domains leads to improvements in the global controlled system performance that could not be pursued by a reliability formulation with fixed failure domains. In addition, the integrated use of asymptotic approximations and deterministic sampling in a hybrid approach proved to considerably relax the high computational demands of the synthesis algorithm. Examples of attitude control of a simple satellite model and disturbance rejection of a flexible beam are used to demonstrate the method.

\section{References}

${ }^{1}$ Crespo, L. G., "Optimal performance, robustness and reliability based designs of systems with structured uncertainty," Proceedings of American Control Conference, Vol. 5, Denver, CO USA, June 2003, pp. 4219-4224.

${ }^{2}$ Laughlin, D. L., Jordan, K. G., and Morari, M., "Internal model control and process uncertainty mapping uncertainty regions for SISO controller design," International Journal of Control, Vol. 44, No. 6, December 1986, pp. 1675-1698.

${ }^{3}$ Zhou, K. and Doyle, J. C., Essentials of Robust Control, Prentice Hall, Upper saddle, New Jersey, 1998.

${ }^{4}$ Marrison, C. and Stengel, R., "Design of Robust Control Systems for a Hypersonic Aircraft," Journal of Guidance, Control, and Dynamics, Vol. 21, January-February 1998, pp. 58-63.

${ }^{5}$ Wang, Q. and Stengel, R. F., "Robust Nonlinear Control of a Hypersonic Aircraft," Journal of Guidance, Control, and Dynamics, Vol. 23, No. 4, July-August 2000, pp. 577-585.

${ }^{6}$ Wang, Q. and Stengel, R. F., "Robust control of nonlinear systems with parametric uncertainty," Automatica, Vol. 38, 2002, pp. 1591-1599.

${ }^{7}$ Crespo, L. G. and Kenny, S. P., "Reliability-based control design for uncertain systems," AIAA Journal of Guidance, Control, and Dynamics, Vol. 28, No. 4, 2005.

${ }^{8}$ Polyak, B. and Tempo, R., "Probabilistic robust design with linear quadratic regulators," Systems and Control Letters, Vol. 43, 2001, pp. 343-353.

${ }^{9}$ Lagoa, C. M., Li, X., and Sznaier, M., "On the Design of Robust Controllers for Arbitrary Uncertainty Structures," IEEE Transactions on Automatic Control, Vol. 48, No. 11, November 2003, pp. 2061-2065.

${ }^{10}$ Spencer, B. F., Sain, M. K., Won, C.-H., Kaspari, D. C., and Sain, P. M., "Reliability-based measures of structural control robustness," Structural Safety, Vol. 15, 1994, pp. 111-129.

${ }^{11}$ Rackwitz, R., "Reliability analysis, a review and some perspectives," Structural Safety, Vol. 23, 2001, pp. 365-395.

${ }^{12}$ May, B. S., Probabilistic Robust Control: Theory and Applications, Ph.D. Dissertation, California Institute of Technology, Pasadena, CA USA, 1997. 
${ }^{13}$ Crespo, L. G., "Probabilistic formulations to robust optimal control," 45th AIAA Structures, Structural Dynamics and Materials Conference, Palm Springs, CA USA, April 2005, pp. 1-21, AIAA Paper No. 20041667 .

${ }^{14}$ Skogestad, S. and Postlethwaite, I., Multivariable feedback control, John Wiley and Sons, Chichester, England, 1996.

${ }^{15}$ Wang, Q. and Stengel, R. F., "Searching for Robust Minimal-Order Compensators," Journal of Dynamic Systems, Measurement and Control, Vol. 123, June 2001, pp. 233-236.

${ }^{16}$ Hokayem, P., Abdallah, C., and Dorato, P., "Quasi-Monte Carlo Methods in Robust Control Design," IEEE Conference on Decision and Control, Maui, HA USA, December 2003, pp. 2435-2440.

${ }^{17}$ Kalagnanam, J. R. and Diwekar, U. M., "An Efficient Sampling Technique for Off-line Quality Control," Technometrics, Vol. 39, No. 3, August 1997, pp. 308-319.

${ }^{18}$ Diwekar, U. M. and Kalagnanam, J. R., "Efficient Sampling Technique for Optimization under Uncertainty," American Institute of Chemical Engineering Journal, Vol. 43, No. 2, February 1997, pp. 440-447.

${ }^{19}$ Kenny, S. P., Optimal rejection of nonstationary narrowband disturbances for flexible systems, Ph.D. thesis, Massachusetts Institute of Technology, Cambridge, MA USA, February, 2002. 